\title{
New Exact Solutions for a Generalized Double Sinh-Gordon Equation
}

\author{
Gabriel Magalakwe and Chaudry Masood Khalique \\ International Institute for Symmetry Analysis and Mathematical Modelling, Department of Mathematical Sciences, \\ North-West University, Mafikeng Campus, Private Bag X 2046, Mmabatho 2735, South Africa
}

Correspondence should be addressed to Chaudry Masood Khalique; masood.khalique@nwu.ac.za

Received 26 June 2013; Revised 15 July 2013; Accepted 15 July 2013

Academic Editor: Santanu Saha Ray

Copyright (C) 2013 G. Magalakwe and C. M. Khalique. This is an open access article distributed under the Creative Commons Attribution License, which permits unrestricted use, distribution, and reproduction in any medium, provided the original work is properly cited.

We study a generalized double sinh-Gordon equation, which has applications in various fields, such as fluid dynamics, integrable quantum field theory, and kink dynamics. We employ the Exp-function method to obtain new exact solutions for this generalized double sinh-Gordon equation. This method is important as it gives us new solutions of the generalized double sinh-Gordon equation.

\section{Introduction}

It is well known that finding exact travelling wave solutions of nonlinear partial differential equations (NLPDEs) is useful in many scientific applications such as fluid mechanics, plasma physics, and quantum field theory. Due to these applications many researchers are investigating exact solutions of NLPDEs since they play a vital role in the study of nonlinear physical phenomena. Finding exact solutions of such NLPDEs provides us with a better understanding of the physical phenomena that these NLPDEs describe. Several techniques have been presented in the literature to find exact solutions of the NLPDEs. These include the homogeneous balance method, the Weierstrass elliptic function expansion method, the F-expansion method, the $\left(G^{\prime} / G\right)$-expansion method, the Exp-function method, the tanh function method, the extended tanh function method, and the Lie group method [1-10].

In this work, we study one such NLPDE, namely, the generalized double sinh-Gordon equation:

$$
u_{t t}-k u_{x x}+2 \alpha \sinh (n u)+\beta \sinh (2 n u)=0, \quad n \geq 1,
$$

which appears in many scientific applications [11-13]. It should be noted that when $k=a, \alpha=(1 / 2) b$, and $\beta=0$,
(1) becomes the generalized sinh-Gordon equation [14, 15]. Furthermore, if $n=a=1$ and $b=2$, (1) reduces to the sinhGordon equation [16].

Many authors have studied the generalized double sinhGordon equation (1). Travelling waves solutions of (1) were obtained in [11] by using the tanh function method and the variable separable method. In [12] the method of bifurcation theory of dynamical system was used to prove the existence of periodic wave, solitary wave, kink and antikink wave, and unbounded wave solutions of (1). It should be noted that solutions obtained in [12] were different the ones obtained in [11]. Recently, solitary and periodic waves solutions of (1) were found in [13] by employing $\left(G^{\prime} / G\right)$-expansion method. It is further shown in [13] that solutions obtained by using the $\left(G^{\prime} / G\right)$-expansion method are more general than those given in [11], which were obtained by tanh function method.

In this paper, we employ an entirely different method, known as the Exp-function method, to obtain new exact solutions of the generalized sinh-Gordon equation (1). The paper is structured as follows. In Section 2, we obtain exact solutions of the generalized double sinh-Gordon equation (1) with the help of the Exp-function method. In Section 3 we present concluding remarks. 


\section{Exact Solutions of (1) Using Exp-Function Method}

In this section we employ the Exp-function method to solve the generalized double sinh-Gordon equation (1). This method was introduced by $\mathrm{He}$ and $\mathrm{Wu}$ [17]. The Exp-function method results in the travelling wave solution based on the assumption that the solution can be expressed in the following form:

$$
H(z)=\frac{\sum_{n=-c}^{d} a_{n} \exp (n z)}{\sum_{m=-p}^{q} b_{m} \exp (m z)}
$$

where $c, d, p$, and $q$ are positive integers that can be determined and $a_{n}$ and $b_{m}$ are unknown constants. According to Exp-function method, we introduce the travelling wave substitution $u(x, t)=W(z)$, where $z=x-c t$. Then (1) transforms to the nonlinear ordinary differential equation:

$$
\left(c^{2}-k\right) W^{\prime \prime}(z)+2 \alpha \sinh (n W(z))+\beta \sinh (2 n W(z))=0 .
$$

Further, using the transformation $W(z)=(1 / n) \ln (H(z))$ on (3), we obtain

$$
\begin{gathered}
2\left(c^{2}-k\right) H(z) H^{\prime \prime}(z)-2\left(c^{2}-k\right) H^{\prime}(z)^{2}+2 \alpha n H(z)^{3} \\
-2 \alpha n H(z)+\beta n H(z)^{4}-\beta n=0 .
\end{gathered}
$$

We assume that the solution of (4) can be expressed as

$$
H(z)=\frac{a_{c} \exp (c z)+\cdots+a_{-d} \exp (-d z)}{b_{p} \exp (p z)+\cdots+b_{-q} \exp (-q z)} .
$$

The values of $c$ and $d, p$ and $q$ can be determined by balancing the linear term of the highest order with the highest order of nonlinear term in (4), that is, $H H^{\prime \prime}$ and $H^{4}$. By straight forward calculation, we have

$$
\begin{gathered}
H H^{\prime \prime}=\frac{c_{1} \exp [(2 c+3 p) z]+\cdots}{c_{2} \exp [5 p z]+\cdots}, \\
H^{4}=\frac{c_{3} \exp [4 c z]+\cdots}{c_{4} \exp [4 p z]+\cdots}=\frac{c_{3} \exp [(4 c+p) z]+\cdots}{c_{4} \exp [5 p z]+\cdots},
\end{gathered}
$$

where $c_{i}$ are coefficients only for simplicity. Balancing the highest order of Exp-function in (6), we have $2 c+3 p=4 c+p$, which yields $c=p$. Similarly, we balance the lowest order in (4) to determine values of $d$ and $q$. We have

$$
\begin{gathered}
H H^{\prime \prime}=\frac{\cdots+s_{1} \exp [-(2 d+3 q) z]}{\cdots+s_{2} \exp [-5 q z]} \\
H^{4}=\frac{\cdots+s_{3} \exp [4 d z]}{\cdots+s_{4} \exp [-4 q z]}=\frac{\cdots+s_{3} \exp [-(4 d+q) z]}{\cdots+s_{4} \exp [-5 q z]}
\end{gathered}
$$

where $s_{i}$ are coefficients only for simplicity. Balancing the lowest order of Exp-function in (7), we have $2 d+3 q=4 d+q$, which yields $d=q$. For simplicity, we first set $c=p=1$ and $d=q=1$. then (5) reduces to

$$
H(z)=\frac{a_{1} \exp (z)+a_{0}+a_{-1} \exp (-z)}{b_{1} \exp (z)+b_{0}+b_{-1} \exp (-z)}
$$

Inserting (8) into (4) and using Maple, we obtain

$$
\begin{aligned}
\frac{1}{B} & {\left[C_{4} \exp (4 z)+C_{3} \exp (3 z)+C_{2} \exp (2 z)\right.} \\
& +C_{1} \exp (z)+C_{0}+C_{-1} \exp (-z) \\
& \left.+C_{-2} \exp (-2 z)+C_{-3} \exp (-3 z)+C_{-4} \exp (-4 z)\right]=0
\end{aligned}
$$

where

$$
\begin{aligned}
& B=\left(b_{1} \exp (z)+b_{0}+b_{-1} \exp (-z)\right)^{4}, \\
& C_{4}=2 \alpha a_{1}^{3} b_{1} n-\beta b_{1}^{4} n+\beta a_{1}^{4} n-2 \alpha a_{1} b_{1}^{3} n, \\
& C_{3}=-2 a_{1}^{2} b_{0} b_{1} c^{2}+2 a_{1} a_{0} b_{1}^{2} c^{2}+6 \alpha a_{0} a_{1}^{2} b_{1} n \\
& -6 \alpha a_{1} b_{0} b_{1}^{2} n+2 a_{1}^{2} b_{0} b_{1} k-2 a_{0} a_{1} b_{1}^{2} k \\
& +2 \alpha a_{1}^{3} b_{0} n-2 a_{0} a_{1} b_{1}^{2} k+2 \alpha a_{1}^{3} b_{0} n \\
& +4 \beta a_{0} a_{1}^{3} n-2 \alpha a_{0} b_{1}^{3} n-4 \beta b_{0} b_{1}^{3} n, \\
& C_{2}=4 \beta a_{-1} a_{1}^{3} n-8 a_{1}^{2} b_{-1} b_{1} c^{2}+8 a_{-1} a_{1} b_{1}^{2} c^{2} \\
& +8 a_{1}^{2} b_{-1} b_{1} k-8 a_{-1} a_{1} b_{1}^{2} k+2 \alpha a_{1}^{3} b_{-1} n \\
& -2 \alpha a_{-1} b_{1}^{3} n-4 \beta b_{-1} b_{1}^{3} n+6 \alpha a_{0} a_{1}^{2} b_{0} n \\
& +6 \alpha a_{0}^{2} a_{1} b_{1} n-6 \alpha a_{1} b_{0}^{2} b_{1} n-6 \beta b_{0}^{2} b_{1}^{2} n \\
& +6 \alpha a_{-1} a_{1}^{2} b_{1} n-6 \alpha a_{1} b_{1}^{2} b_{-1} n+6 \beta a_{0}^{2} a_{1}^{2} n \\
& -6 \alpha a_{0} b_{0} b_{1}^{2} n \\
& C_{1}=-2 a_{0}^{2} b_{0} b_{1} c^{2}+2 a_{0} a_{1} b_{0}^{2} c^{2}+2 a_{0}^{2} b_{0} b_{1} k \\
& -2 a_{0} a_{1} b_{0}^{2} k-2 a_{1}^{2} b_{0} b_{-1} c^{2}+2 a_{-1} a_{0} b_{1}^{2} c^{2} \\
& -2 a_{0} a_{-1} b_{1}^{2} k+2 \alpha a_{0}^{3} b_{1} n+4 \beta a_{0}^{3} a_{1} n-2 \alpha a_{1} b_{0}^{3} n \\
& -4 \beta b_{0}^{3} b_{1} n+12 a_{-1} a_{1} b_{0} b_{1} c^{2}-12 a_{-1} a_{1} b_{0} b_{1} k \\
& +12 a_{0} a_{1} b_{-1} b_{1} k+6 \alpha a_{0}^{2} a_{1} b_{0} n-6 \alpha a_{0} b_{0}^{2} b_{1} n \\
& +12 \alpha a_{-1} a_{0} a_{1} b_{1} n-12 \alpha a_{1} b_{-1} b_{0} b_{1} n \\
& +6 \alpha a_{-1} a_{1}^{2} b_{0} n-6 \alpha a_{0} b_{-1} b_{1}^{2} n-6 \alpha a_{-1} b_{0} b_{1}^{2} n \\
& +6 \alpha a_{0} a_{1}^{2} b_{-1} n+12 \beta a_{-1} a_{0} a_{1}^{2} n-12 \beta b_{-1} b_{0} b_{1}^{2} n \\
& +2 a_{1}^{2} b_{0} b_{-1} k-12 a_{0} a_{1} b_{-1} b_{1} c^{2} \text {, }
\end{aligned}
$$




$$
\begin{aligned}
& C_{0}=2 \alpha a_{0}^{3} b_{0} n-2 \alpha a_{0} b_{0}^{3} n+\beta a_{0}^{4} n \\
& +6 \alpha a_{-1} a_{1}^{2} b_{-1} n+6 \alpha a_{0}^{2} a_{1} b_{-1} n+6 \alpha a_{-1}^{2} a_{1} b_{1} n \\
& +6 \alpha a_{-1} a_{0}^{2} b_{1} n+12 \beta a_{-1} a_{0}^{2} a_{1} n-6 \alpha a_{1} b_{-1}^{2} b_{1} n \\
& -6 \alpha a_{1} b_{-1} b_{0}^{2} n-6 \alpha a_{-1} b_{-1} b_{1}^{2} n-\beta b_{0}^{4} n \\
& -6 \alpha a_{-1} b_{0}^{2} b_{1} n-12 \beta b_{-1} b_{0}^{2} b_{1} n+8 a_{-1} a_{1} b_{0}^{2} c^{2} \\
& -8 a_{0}^{2} b_{-1} b_{1} c^{2}-8 a_{-1} a_{1} b_{0}^{2} k+8 a_{0}^{2} b_{-1} b_{1} k \\
& +6 \beta a_{-1}^{2} a_{1}^{2} n-6 \beta b_{-1}^{2} b_{1}^{2} n+12 \alpha a_{-1} a_{0} a_{1} b_{0} n \\
& -12 \alpha a_{0} b_{-1} b_{0} b_{1} n \text {, } \\
& C_{-1}=12 \alpha a_{-1} a_{0} a_{1} b_{-1} n-12 \alpha a_{-1} b_{-1} b_{0} b_{1} n \\
& +2 a_{-1} a_{0} b_{0}^{2} c^{2}-2 a_{0}^{2} b_{-1} b_{0} c^{2}+2 a_{0}^{2} b_{-1} b_{0} k \\
& -2 a_{-1} a_{0} b_{0}^{2} k+2 a_{0} a_{1} b_{-1}^{2} c^{2}-2 a_{-1}^{2} b_{0} b_{1} c^{2} \\
& -2 a_{0} a_{1} b_{-1}^{2} k+2 a_{-1}^{2} b_{0} b_{1} k+2 \alpha a_{0}^{3} b_{-1} n \\
& +4 \beta a_{-1} a_{0}^{3} n-2 \alpha a_{-1} b_{0}^{3} n-4 \beta b_{-1} b_{0}^{3} n \\
& +12 a_{-1} a_{1} b_{-1} b_{0} c^{2}-12 a_{-1} a_{0} b_{-1} b_{1} c^{2} \\
& -12 a_{-1} a_{1} b_{-1} b_{0} k+12 a_{-1} a_{0} b_{-1} b_{1} k \\
& +6 \alpha a_{-1} a_{0}^{2} b_{0} n-6 \alpha a_{0} b_{-1} b_{0}^{2} n+6 \alpha a_{-1}^{2} a_{1} b_{0} n \\
& +6 \alpha a_{-1}^{2} a_{0} b_{1} n+12 \beta a_{-1}^{2} a_{0} a_{1} n-6 \alpha a_{1} b_{-1}^{2} b_{0} n \\
& -6 \alpha a_{0} b_{-1}^{2} b_{1} n-12 \beta b_{-1}^{2} b_{0} b_{1} n, \\
& C_{-2}=2 \alpha a_{-1}^{3} b_{1} n+8 a_{-1} a_{1} b_{-1}^{2} c^{2}+8 a_{-1}^{2} b_{-1} b_{1} k \\
& -8 a_{-1} a_{1} b_{-1}^{2} k+4 \beta a_{-1}^{3} a_{1} n-4 \beta b_{-1}^{3} b_{1} n \\
& -2 \alpha a_{1} b_{-1}^{3} n-8 a_{-1}^{2} b_{-1} b_{1} c^{2}+6 \alpha a_{-1} a_{0}^{2} b_{-1} n \\
& +6 \alpha a_{-1}^{2} a_{0} b_{0} n-6 \alpha a_{0} b_{-1}^{2} b_{0} n+6 \alpha a_{-1}^{2} a_{1} b_{-1} n \\
& -6 \alpha a_{-1} b_{-1}^{2} b_{1} n+6 \beta a_{-1}^{2} a_{0}^{2} n-6 \beta b_{-1}^{2} b_{0}^{2} n \\
& -6 \alpha a_{-1} b_{-1} b_{0}^{2} n \text {, } \\
& C_{-3}=6 \alpha a_{0} a_{-1}^{2} b_{-1} n-6 \alpha a_{-1} b_{-1}^{2} b_{0} n \\
& -2 a_{-1}^{2} b_{-1} b_{0} c^{2}+2 a_{-1} a_{0} b_{-1}^{2} c^{2} \\
& +2 a_{-1}^{2} b_{0} b_{-1} k-2 a_{-1} a_{0} b_{-1}^{2} k \\
& +2 \alpha a_{-1}^{3} b_{0} n+4 \beta a_{0} a_{-1}^{3} n \\
& -2 \alpha a_{0} b_{-1}^{3} n-4 \beta b_{0} b_{-1}^{3} n \\
& C_{-4}=\beta a_{-1}^{4} n-\beta b_{-1}^{4} n+2 \alpha a_{-1}^{3} b_{-1} n-2 \alpha a_{-1} b_{-1}^{3} n \text {. }
\end{aligned}
$$

Equating the coefficients of $\exp (z)$ in (9) to zero, we obtain a set of algebraic equations:

$$
\begin{aligned}
& C_{4}=0, \quad C_{3}=0, \quad C_{2}=0, \quad C_{1}=0, \quad C_{0}=0, \\
& C_{-1}=0, \quad C_{-2}=0, \quad C_{-3}=0, \quad C_{-4}=0 .
\end{aligned}
$$

Solving the system (11) with the help of Maple, we obtain the following three cases.

Case 1. We have the following:

$$
\begin{gathered}
a_{-1}=b_{-1}, \quad a_{0}=-b_{0}, \quad a_{1}=b_{1}, \quad \beta=\frac{\alpha b_{0}^{2}-4 \alpha b_{1} b_{-1}}{4 b_{1} b_{-1}}, \\
k=\frac{\alpha b_{0}^{2} n+2 b_{-1} b_{1} c^{2}}{2 b_{-1} b_{1}} .
\end{gathered}
$$

Case 2. We have the following:

$$
\begin{gathered}
a_{-1}=\frac{b_{-1} b_{1}}{a_{1}}, \quad a_{0}=0, \quad b_{0}=0, \quad \alpha=\frac{-\beta\left(a_{1}^{2}+b_{1}^{2}\right)}{2 a_{1} b_{1}}, \\
k=\frac{-2 \beta a_{1}^{2} b_{1}^{2} n+\beta a_{1}^{4} n+\beta b_{1}^{4} n+8 a_{1}^{2} b_{1}^{2} c^{2}}{8 a_{1}^{2} b_{1}^{2}} .
\end{gathered}
$$

Case 3. We have the following:

$$
\begin{gathered}
a_{-1}=-\phi b_{1}, \quad b_{-1}=-\phi a_{1}, \quad \alpha=\frac{-\beta\left(a_{1}^{2}+b_{1}^{2}\right)}{2 a_{1} b_{1}}, \\
k=\frac{-2 \beta a_{1}^{2} b_{1}^{2} n+\beta a_{1}^{4} n+\beta b_{1}^{4} n+2 a_{1}^{2} b_{1}^{2} c^{2}}{2 a_{1}^{2} b_{1}^{2}}
\end{gathered}
$$

where $\phi=\left(-a_{0} a_{1}^{2} b_{0}+a_{0}^{2} a_{1} b_{1}+a_{1} b_{0}^{2} b_{1}-a_{0} b_{0} b_{1}^{2}\right) /\left(a_{1}-b_{1}\right)^{2}\left(a_{1}+\right.$ $\left.b_{1}\right)^{2}$.

Substituting values from (12) into (8), we obtain

$$
H(z)=\frac{b_{1} \exp (z)-b_{0}+b_{-1} \exp (-z)}{b_{1} \exp (z)+b_{0}+b_{-1} \exp (-z)} .
$$

As a result one of the solutions of (1) is given by

$$
u_{1}(x, t)=\frac{1}{n} \ln \left(\frac{b_{1} \exp (z)-b_{0}+b_{-1} \exp (-z)}{b_{1} \exp (z)+b_{0}+b_{-1} \exp (-z)}\right),
$$

where $z=x-c t, \beta=\left(\alpha b_{0}^{2}-4 \alpha b_{1} b_{-1}\right) / 4 b_{1} b_{-1}$, and $k=\left(\alpha b_{0}^{2} n+\right.$ $\left.2 b_{-1} b_{1} c^{2}\right) / 2 b_{-1} b_{1}$.

As a special case, if we choose $b_{0}=2$ and $b_{-1}=b_{1}=1$ in (16), then we get $\beta=0, k=2 \alpha n+c^{2}$ and obtain the solution of the generalized sinh-Gordon equation as

$$
u_{1}(x, t)=\frac{1}{n} \ln \left(\tanh ^{2}\left[\left(\frac{1}{2}\right)(x-c t)\right]\right),
$$

which is the solution obtained in $[14,15]$. 
Now substituting the values from (13) (Case 2) into (8) results in the second solution of (1) as

$$
u_{2}(x, t)=\frac{1}{n} \ln \left(\frac{a_{1} \exp (z)+\left(b_{-1} b_{1} / a_{1}\right) \exp (-z)}{b_{1} \exp (z)+b_{-1} \exp (-z)}\right) \text {, }
$$

with $z=x-c t, \alpha=-\beta\left(a_{1}^{2}+b_{1}^{2}\right) / 2 a_{1} b_{1}$, and $k=\left(-2 \beta a_{1}^{2} b_{1}^{2} n+\right.$ $\left.\beta a_{1}^{4} n+\beta b_{1}^{4} n+8 a_{1}^{2} b_{1}^{2} c^{2}\right) / 8 a_{1}^{2} b_{1}^{2}$.

The third solution of (1) is obtained by using the values from (14) (Case 3) and substituting them into (8). Consequently, it is given by

$$
u_{3}(x, t)=\frac{1}{n} \ln \left(\frac{a_{1} \exp (z)+a_{0}-b_{1} \phi \exp (-z)}{b_{1} \exp (z)+b_{0}-a_{-1} \phi \exp (-z)}\right),
$$

where $z=x-c t, \phi=\left(-a_{0} a_{1}^{2} b_{0}+a_{0}^{2} a_{1} b_{1}+a_{1} b_{0}^{2} b_{1}-a_{0} b_{0} b_{1}^{2}\right) /\left(a_{1}-\right.$ $\left.b_{1}\right)^{2}\left(a_{1}+b_{1}\right)^{2}, \alpha=-\beta\left(a_{1}^{2}+b_{1}^{2}\right) / 2 a_{1} b_{1}$, and $k=\left(-2 \beta a_{1}^{2} b_{1}^{2} n+\right.$ $\left.\beta a_{1}^{4} n+\beta b_{1}^{4} n+2 a_{1}^{2} b_{1}^{2} c^{2}\right) / 2 a_{1}^{2} b_{1}^{2}$.

To construct more solutions of (1), we now set $c=p=2$ and $d=q=2$. Then (5) reduces to

$$
\begin{aligned}
H(z)= & \left(a_{2} \exp (2 z)+a_{1} \exp (z)+a_{0}+a_{-1} \exp (-z)\right. \\
& \left.+a_{-2} \exp (-2 z)\right) \\
& \times\left(b_{2} \exp (z)+b_{1} \exp (z)+b_{0}\right. \\
& \left.+b_{-1} \exp (-z)+b_{-2} \exp (-2 z)\right)^{-1}
\end{aligned}
$$

Proceeding as above, we obtain the following three solutions of (1):

$$
\begin{gathered}
u_{4}(x, t)=\frac{1}{n} \ln \left(a_{2} \exp (2 z)+\left(\frac{a_{-1} b_{1}}{b_{-1}}\right) \exp (z)\right. \\
\left.\quad+\left(\frac{a_{-1} b_{0}}{b_{-1}}\right)+a_{-1} \exp (-z)\right) \\
\times\left(\frac{a_{2} b_{-1}}{a_{-1}} \exp (z)+b_{1} \exp (z)\right. \\
\left.+b_{0}+b_{-1} \exp (-z)\right)^{-1},
\end{gathered}
$$

where $z=x-c t, \alpha=-\beta\left(a_{-1}^{2}+b_{-1}^{2}\right) / 2 a_{-1} b_{-1}$,

$$
u_{5}(x, t)=\frac{1}{n} \ln \left(\frac{a_{2} \exp (2 z)+a_{1} \exp (z)+b_{0}}{-a_{2} \exp (z)+b_{1} \exp (z)+b_{0}}\right) \text {, }
$$

with $z=x-c t, \beta=\alpha\left(b_{1}^{2}+4 a_{2} b_{0}\right) / 4 a_{2} b_{0}$, and $k=\left(\alpha n b_{1}^{2}+\right.$ $\left.2 a_{2} b_{0} c^{2}\right) / 2 a_{2} b_{0}$, and

$$
u_{6}(x, t)=\frac{1}{n} \ln \left(\frac{a_{2} \exp (2 z)-b_{0}+b_{-2} \exp (-2 z)}{a_{2} \exp (2 z)+b_{0}+b_{-2} \exp (-2 z)}\right)
$$

where $z=x-c t, \alpha=-\left(8 a_{2} b_{-2}\left(c^{2}-k\right) / b_{0}^{2} n\right)$, and $\beta=$ $2\left(4 a_{2} b_{-2} c^{2}-4 a_{2} b_{-2} k-b_{0}^{2} c^{2}+b_{0}^{2} k\right) / b_{0}^{2} n$.

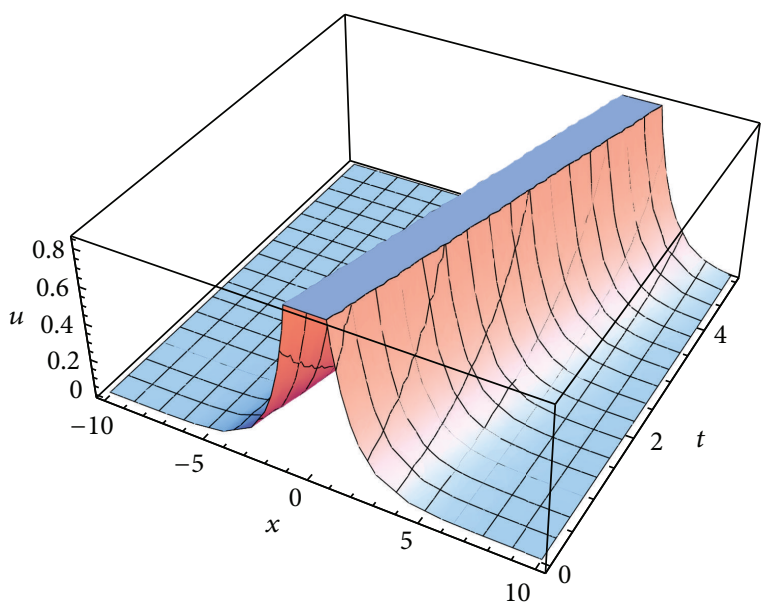

FIgURE 1: Profile of solution (16).

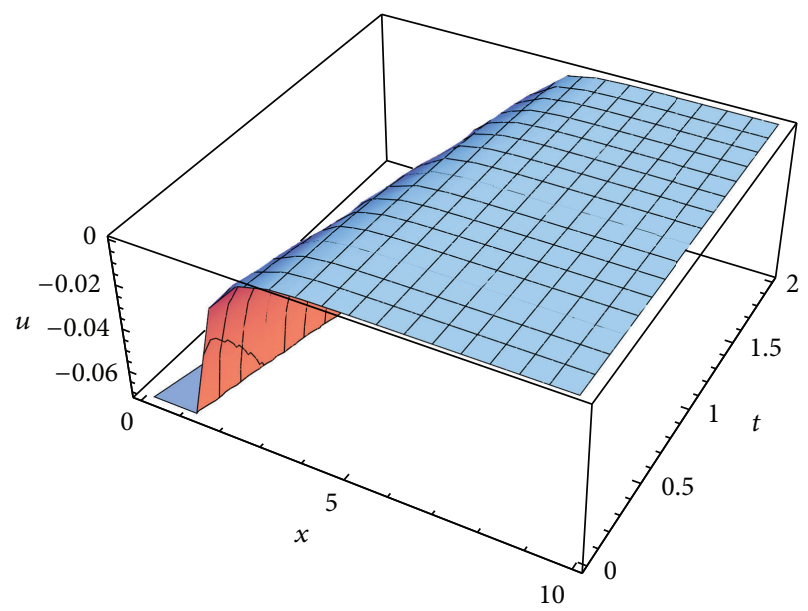

FIgURE 2: Profile of solution (23).

By taking $n=2, b_{-1}=-1, b_{0}=2, c=1$, and $b_{1}=-1$ in the solution (16), we have its profile given in Figure 1 .

By taking $n=3, b_{-2}=1, b_{0}=2, c=1$, and $a_{1}=1$ in the solution (23), we have its profile given in Figure 2.

\section{Concluding Remarks}

In this paper we obtained new exact solutions of the generalized double sinh-Gordon equation (1) using the Expfunction method. We presented six different solutions of (1). Earlier, the tanh function, the bifurcation, and the $\left(G^{\prime} / G\right)$ expansion methods [11-13] were employed to obtain exact solutions of (1). The solutions obtained in this paper were new and were different from the ones obtained in [11-13]. By taking special values of the constants, we also retrieved the solution of the generalized sinh-Gordon equation, which was obtained in $[14,15]$. The Exp-function method is very simple and straightforward method for solving nonlinear partial differential equations. Indeed this has some pronounced merit as compared to the other methods. The correctness of 
the solutions obtained here has been verified by substituting them back into (1).

\section{Acknowledgments}

Gabriel Magalakwe would like to thank SANHARP, NRF, and North-West University, Mafikeng Campus, South Africa, for their financial support.

\section{References}

[1] M. Wang, Y. Zhou, and Z. Li, "Application of a homogeneous balance method to exact solutions of nonlinear equations in mathematical physics," Physics Letters A, vol. 216, no. 1-5, pp. 67-75, 1996.

[2] Y. Chen and Z. Yan, "The Weierstrass elliptic function expansion method and its applications in nonlinear wave equations," Chaos, Solitons and Fractals, vol. 29, no. 4, pp. 948-964, 2006.

[3] M. Wang and X. Li, "Applications of F-expansion to periodic wave solutions for a new Hamiltonian amplitude equation," Chaos, Solitons and Fractals, vol. 24, no. 5, pp. 1257-1268, 2005.

[4] M. Wang, X. Li, and J. Zhang, “The $\left(G^{\prime}, G\right)$-expansion method and travelling wave solutions of nonlinear evolution equations in mathematical physics," Physics Letters A, vol. 372, no. 4, pp. 417-423, 2008.

[5] S. Zhang, "Application of Exp-function method to highdimensional nonlinear evolution equation," Chaos, Solitons and Fractals, vol. 38, no. 1, pp. 270-276, 2008.

[6] H. Zhi and H. Zhang, "Applications of the combined tanh function method with symmetry method to the nonlinear evolution equations," Applied Mathematics and Computation, vol. 188, no. 1, pp. 385-393, 2007.

[7] D.-S. Li and H.-Q. Zhang, "A further extended tanh-function method and new soliton-like solutions to the integrable BroerKaup (BK) equations in $(2+1)$ dimensional spaces," Applied Mathematics and Computation, vol. 147, no. 2, pp. 537-545, 2004.

[8] G. W. Bluman and S. Kumei, Symmetries and Differential Equations, vol. 81 of Applied Mathematical Sciences, Springer, New York, NY, USA, 1989.

[9] P. J. Olver, Applications of lie Groups to Differential Equations, vol. 107 of Graduate Texts in Mathematics, Springer, Berlin, Germany, 2nd edition, 1993.

[10] N. H. Ibragimov, CRC Handbook of Lie Group Analysis of Differential Equations, vol. 1-3, CRC Press, Boca Raton, Fla, USA, 1994-1996.

[11] A. M. Wazwaz, "Exact solutions to the double sinh-Gordon equation by the tanh method and a variable separated ODE method," Computers and Mathematics with Applications, vol. 50, no. 10-12, pp. 1685-1696, 2005.

[12] S. Tang and W. Huang, "Bifurcations of travelling wave solutions for the generalized double sinh-Gordon equation," Applied Mathematics and Computation, vol. 189, no. 2, pp. 1774-1781, 2007.

[13] H. Kheiri and A. Jabbari, "Exact solutions for the double sinhGordon and the generalized form of the double sinh-Gordon equations by $\left(G^{\prime} / G\right)$-expansion method," Turkish Journal of Physics, vol. 34, no. 2, pp. 73-82, 2010.

[14] A.-M. Wazwaz, "Exact solutions for the generalized sineGordon and the generalized sinh-Gordon equations," Chaos, Solitons and Fractals, vol. 28, no. 1, pp. 127-135, 2006.
[15] K. Parand, J. A. Rad, and A. Rezaei, "Application of Expfunction method for class of nonlinear PDE's in mathematical physics," Journal of Applied Mathematics \& Informatics, vol. 29, pp. 763-779, 2011.

[16] A.-M. Wazwaz, "The tanh method: exact solutions of the sineGordon and the sinh-Gordon equations," Applied Mathematics and Computation, vol. 167, no. 2, pp. 1196-1210, 2005.

[17] J.-H. He and X.-H. Wu, "Exp-function method for nonlinear wave equations," Chaos, Solitons and Fractals, vol. 30, no. 3, pp. 700-708, 2006. 


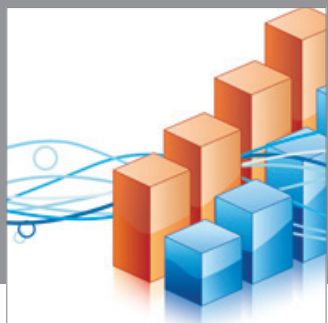

Advances in

Operations Research

mansans

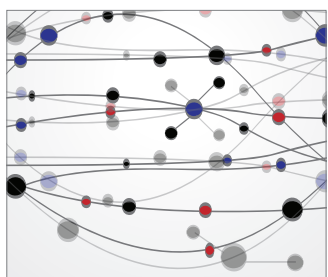

The Scientific World Journal
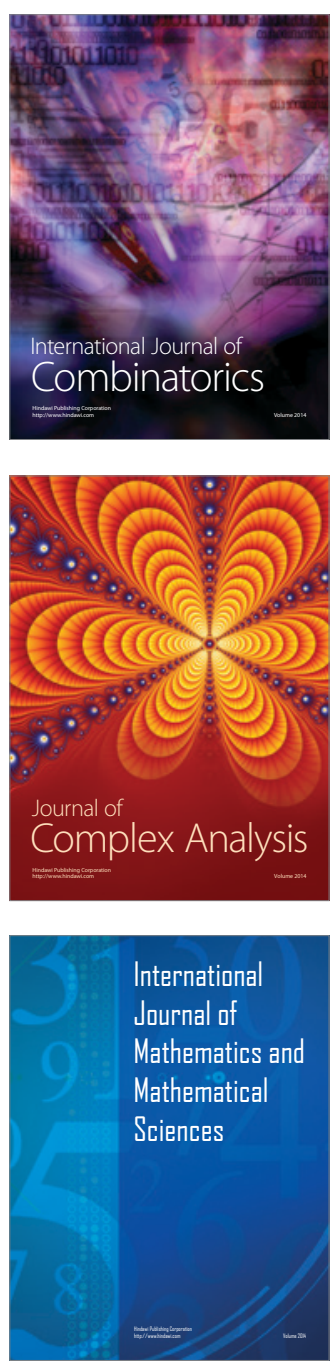
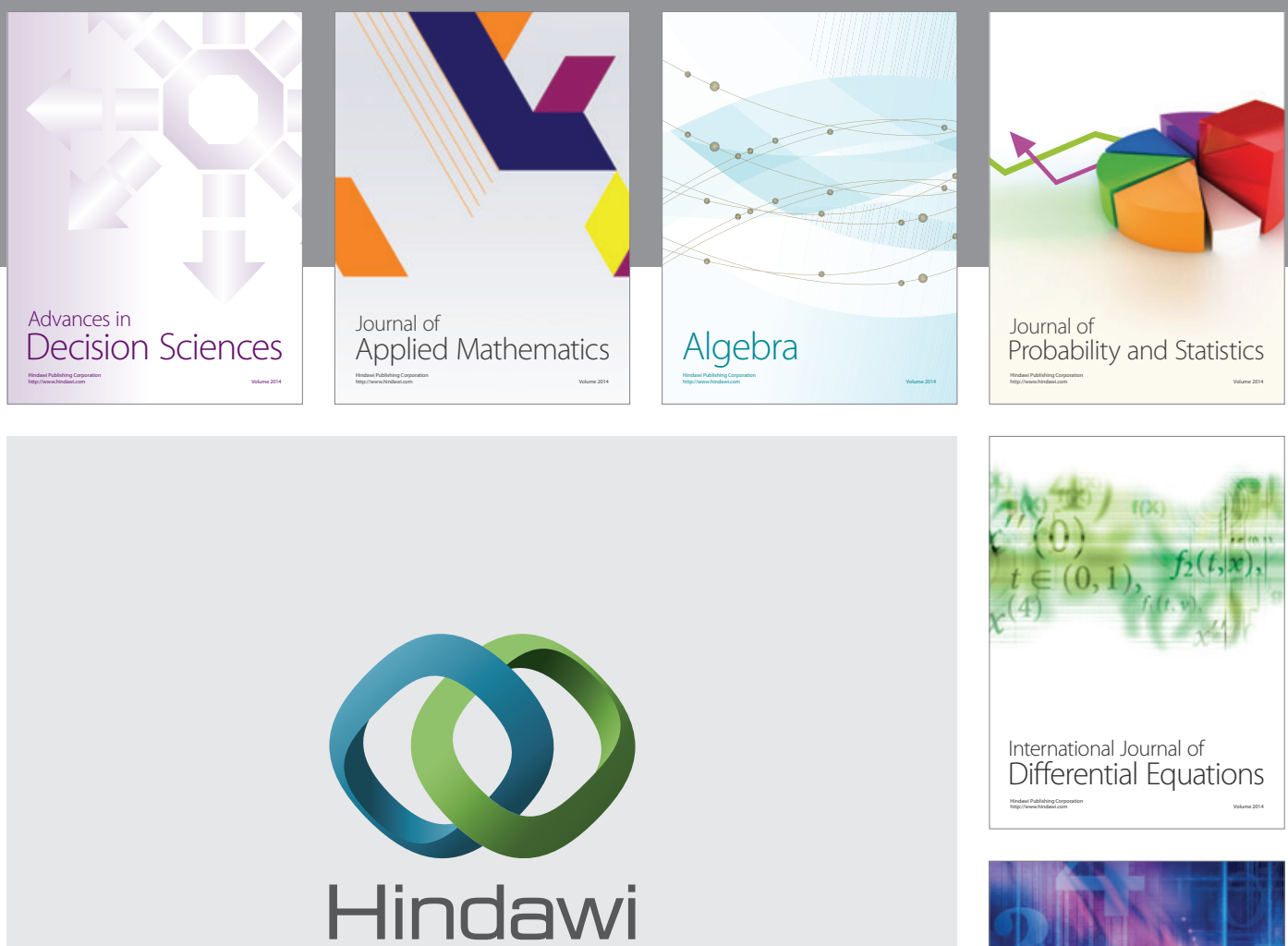

Submit your manuscripts at http://www.hindawi.com
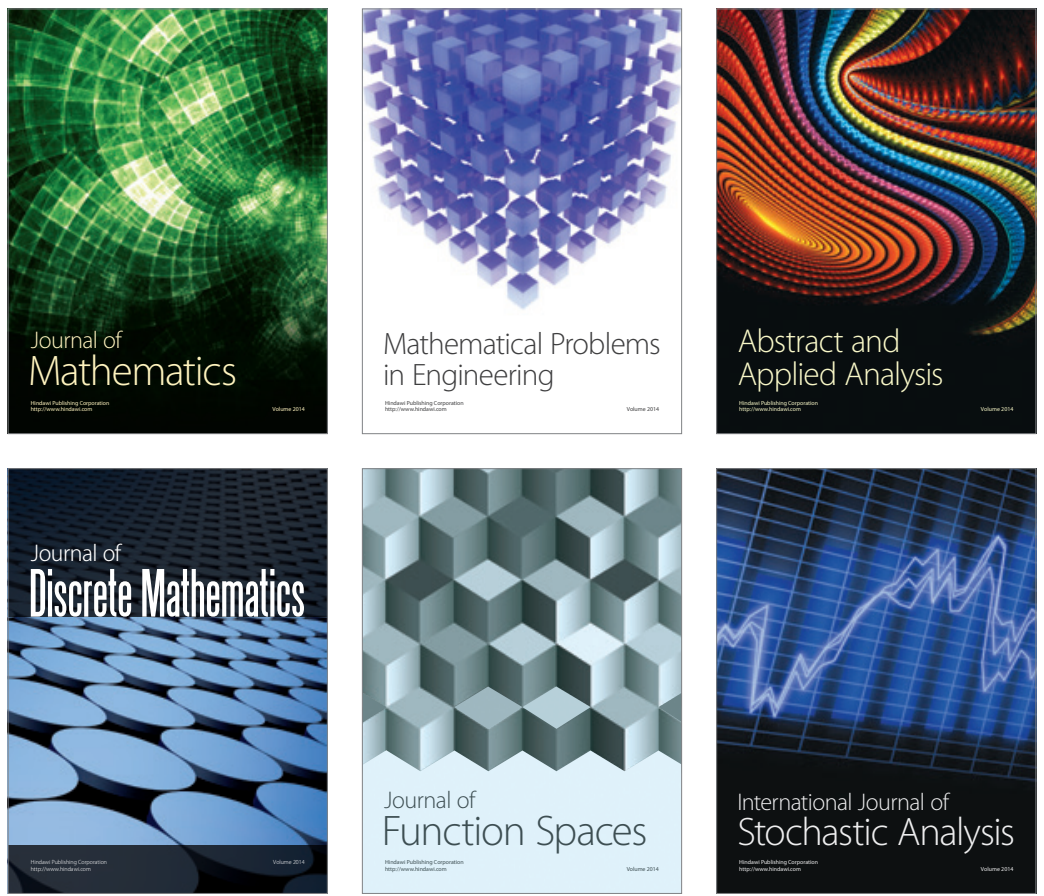

Journal of

Function Spaces

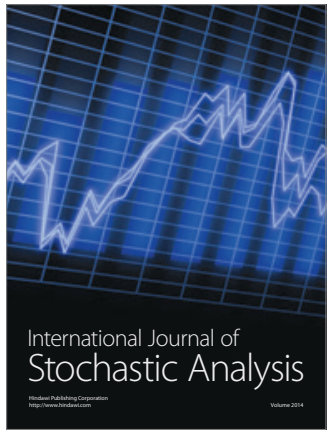

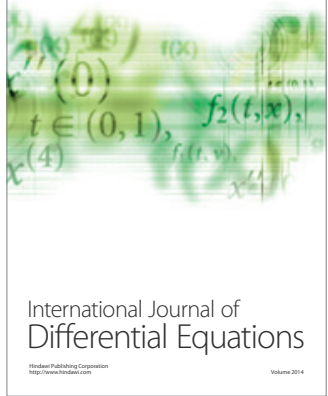
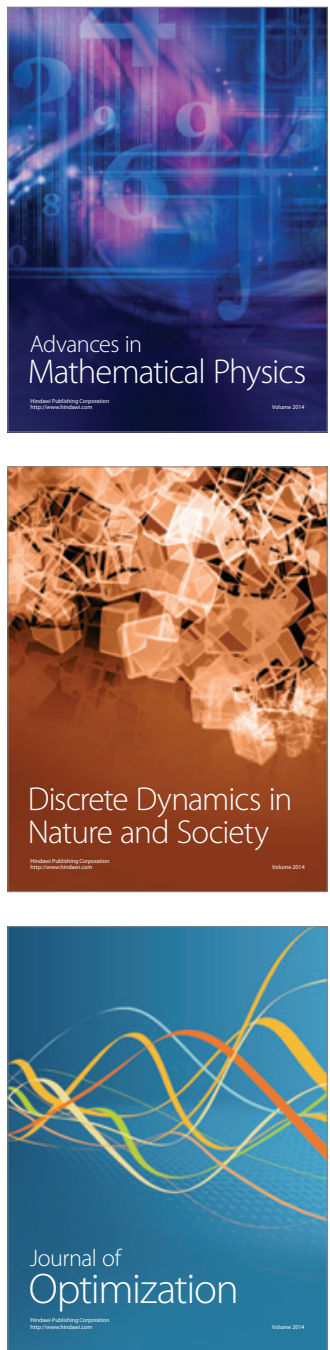